\title{
RELATOS DE PESQUISAS \\ TRANSFORMAÇÕES CONCEITUAIS DE COMUNIDADES DE PRÁTICA: \\ DA APRENDIZAGEM SITUADA À GESTÃO ORGANIZACIONAL
}

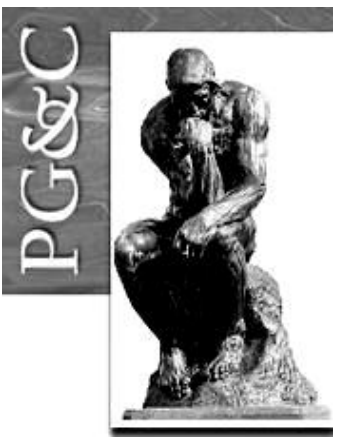

\author{
Julieta Kaoru Watanabe Wilbert \\ Doutoranda em Engenharia e Gestão do Conhecimento pela \\ Universidade Federal de Santa Catarina, Brasil. \\ E-mail: julieta.wilbert@gmx.net
}

Gertrudes Aparecida Dandolini

Doutora em Engenharia da Produção pela Universidade Federal de Santa Catarina, Brasil. Professora da Universidade Federal de Santa Catarina, Brasil. E-mail: Gertrudes.dandolini@egc.ufsc.br

\author{
Andrea Valéria Steil \\ Doutora em Engenharia da Produção pela Universidade Federal de Santa \\ Catarina, Brasil. Professora da Universidade Federal de Santa Catarina, Brasil. \\ E-mail: Andreasteil@egc.ufsc.br
}

\begin{abstract}
Resumo
Comunidades de prática (CoPs) têm sido alvo de pesquisas na área de gestão do conhecimento. Entretanto, a definição de "comunidade de prática" apresenta polissemias. Para compreender o que é CoP, este artigo identifica interpretações acerca dessa expressão a partir de revisão sistemática. Concluiu-se que o conceito de CoP se alterou com o passar do tempo para se adequar aos diversos contextos em que elas encontraram aplicabilidade. Assim, aspectos antes não considerados, como a criação de CoPs pela organização, passaram a ser aceitos. Dessa forma, a partir de um conceito analítico da área da aprendizagem situada, o grande ponto de inflexão conceitual de CoPs foi a sua transformação para um conceito instrumental, onde se descobriu a utilidade do conceito para a aplicação na prática. Os resultados desta pesquisa mostraram que, não obstante as transformações conceituais, há elementos centrais que permanecem intrínsecos ao conceito de CoP, em suas várias abordagens: existência de um domínio como núcleo de atração de pessoas, voluntariedade na adesão, informalidade no funcionamento, existência de empreendimento conjunto, engajamento mútuo e repertório compartilhado. Este artigo traz, adicionalmente, uma síntese da transformação do conceito de CoP relatada por Wenger-Trayner, que junto com Lave (1991) definiu Comunidade de Prática no contexto da academia. O estudo contribui para a compreensão do processo de transformação do entendimento de CoPs ao longo do tempo, e mostra que conceitos são passíveis de adaptações contextuais e temporais.
\end{abstract}

Palavras-chave: CoP. Comunidade de prática. Definição de CoP. Gestão do Conhecimento.

\section{CONCEPTUAL TRANSFORMATIONS OF COMMUNITIES OF PRACTICE: OF LEARNING LOCATED IN ORGANIZATIONAL MANAGEMENT}

\begin{abstract}
Communities of practice (CoPs) have been a research subject in the field of knowledge management. However, the different definitions of "community of practice" exhibit diversities and even contradictions. In order to understand what a COP is, this article presents interpretations about this expression through a
\end{abstract}

Perspectivas em Gestão \& Conhecimento, João Pessoa, v. 8, número especial, p. 102-117, out. 2018. DOI: http://dx.doi.org/10.21714/2236-417X2018v8nep102

http://periodicos.ufpb.br/ojs2/index.php/pgc. ISSN: 2236-417X. Publicação sob Licença (cc) EY-NC-ND 
systematic review. It was concluded that the concept of a CoP changed over time to fit the different contexts in which they found applicability. Thus, the great conceptual inflection of CoPs was its transformation from an analytical concept of the situated learning to an instrumental concept, where the usefulness of the concept for the practical application was discovered. In spite of the conceptual transformations, there are central elements that remain intrinsic to the concept of a CoP in its various approaches: the existence of a domain as a nucleus for the attraction of people, the willingness in the adhesion, the informality in the operation as well as the existence of a joint venture, mutual engagement, and shared repertoire. This article also presents a synthesis of the transformation of the CoP concept reported by Wenger-Trayner, who together with Lave (1991), defined Community of Practice in the context of the academic environment. The study contributes to the understanding of how the definition of CoPs changed over time and demonstrates that concepts are amenable to contextual and temporal adaptations.

Keywords: CoP. Communities of Practice. Definition of CoP. Knowledge Management.

\section{INTRODUÇÃO}

Comunidades de prática (CoPs) e suas variantes mediadas por tecnologia comunidades de prática virtuais (VCoPs) - têm despertado interesse tanto no meio acadêmico, como em consultores, e em organizações privadas e públicas. Aceitas na atualidade como ferramenta ou técnica de gestão do conhecimento, sobretudo em contextos corporativos (YOUNG, 2010), as comunidades de prática são investigadas na academia como apoio à geração de novos conhecimentos, em busca de sua associação com outros fenômenos organizacionais (HILDRETH; KIMBLE, 2004).

Uma análise da literatura sobre comunidades de prática indica a existência de olhares múltiplos para o fenômeno (MENDES; URBINA, 2015). Publicações revelam que são várias as áreas da ciência que investigam CoPs, por exemplo, saúde, educação, gestão organizacional, gestão do conhecimento, sociologia, dentre algumas. Por um lado, a riqueza de perspectivas em analisar comunidades de prática indica que elas são transversais a disciplinas e aplicáveis em vários contextos; por outro lado, essa mesma diversidade gera diferentes entendimentos para um mesmo objeto.

Segundo Wilbert (2015), o conceito de CoP mostra-se polissêmico e dinâmico ao longo do tempo. Tal realidade remete a uma questão central para o desenvolvimento científico cumulativo nessa área: o que são, afinal, comunidades de prática?

O presente artigo visa a investigar possíveis transformações do conceito de CoP ao longo do tempo. A estratégia de investigação utilizada para buscar a resposta foi uma revisão sistemática na base Scopus e Scielo realizada em duas etapas. A primeira etapa, até 2014, visou à elaboração de pesquisa de mestrado da primeira autora. Na segunda etapa realizou-se uma atualização da mesma pesquisa, e assim, uma continuidade no acompanhamento da compreensão do construto "comunidade de prática" em um cenário de transformações contínuas da sociedade.

O presente artigo relata, após a introdução, uma síntese cronológica a partir da revisão da literatura sobre os aspectos relevantes de CoPs para este artigo. Na sequência descrevemse os procedimentos metodológicos, seguidos da apresentação dos resultados e das discussões. Ao final, são tecidas as considerações finais.

Perspectivas em Gestão \& Conhecimento, João Pessoa, v. 8, número especial, p. 102-117, out. 2018. 


\section{REVISÃO DA LITERATURA}

\subsection{Panorama de estudos sobre CoPs em contextos organizacionais}

Com o objetivo de fornecer ao leitor uma visão geral do comportamento das pesquisas publicadas sobre CoPs como objeto de investigação científica, a Figura 1 apresenta dados fornecidos pela base Scopus, desde que nela se iniciaram os registros em artigos e periódicos, com o descritor "comunidade de prática" no título.

Figura 1 - Número de Publicações

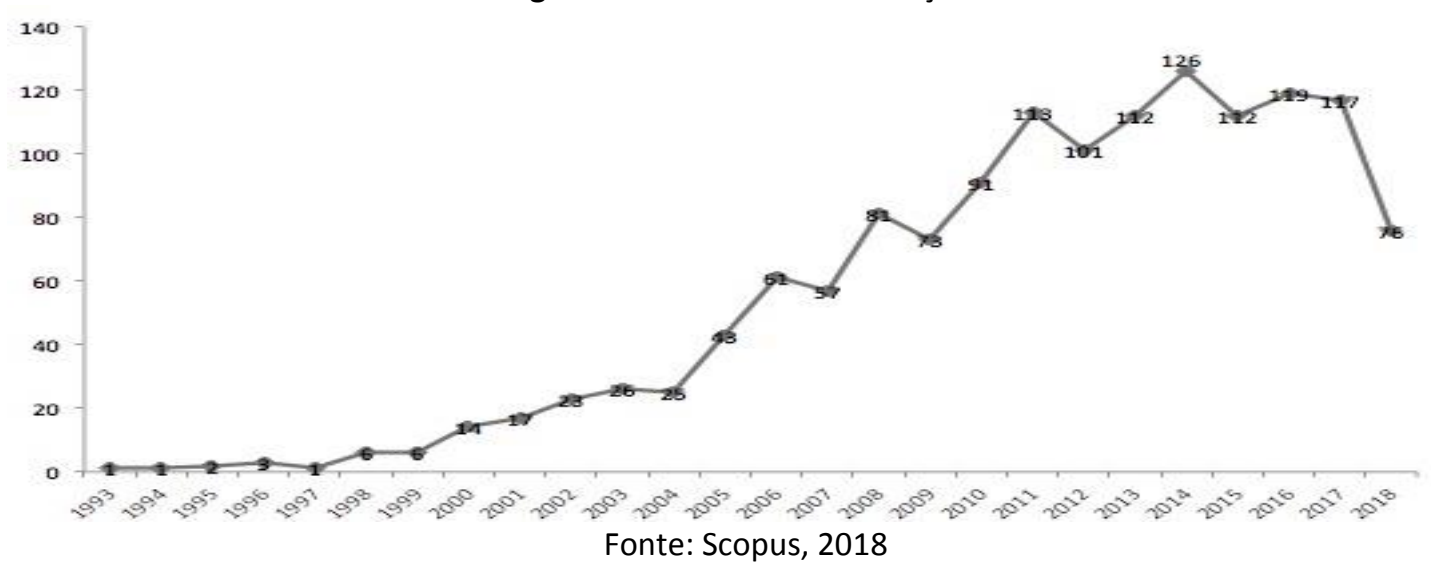

Nesse período de tempo, os cinco periódicos com maior número de publicações com o título "comunidades de prática foram (nesta ordem): Journal of Knowledge Management, International Journal of Web Based Communities, Teaching and Teacher Education, Implementation Science e Journal of Continuing Education in the Health Professions. Essa lista revela que o campo da gestão de conhecimento (portanto, da gestão organizacional) e da educação são promotoras de pesquisas científicas sobre CoPs.

A presença de investigações sobre CoPs em várias áreas do conhecimento enfatiza o caráter multidisciplinar do tema ao longo de décadas (Figura 2).

Figura 2 - CoPs e Áreas do Conhecimento (1993 - 2017)

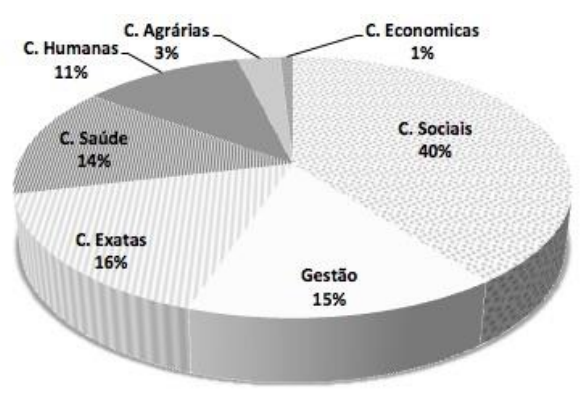

Fonte: Scopus, 2017

Esse cenário torna compreensível a existência de diversos entendimentos e perspectivas para se definir Comunidade de Prática, e por esse motivo, apresenta-se, nesta revisão, uma síntese cronológica da evolução conceitual do construto.

Perspectivas em Gestão \& Conhecimento, João Pessoa, v. 8, número especial, p. 102-117, out. 2018. 
A definição primária de CoP pode ser atribuída a Lave e Wenger (1991), que descrevem a expressão "comunidade de prática" como um "sistema de atividades no qual participantes compartilham entendimentos sobre o que fazem, e o que isso significa em suas vidas e para a sua comunidade" (p. 98). Essa definição foi concebida pelos mencionados autores em um contexto de aprendizagem situada, em que a aprendizagem é fruto de evento social, para além de uma dinâmica psicológica individual (CORSO; GIACOBBE ; MARTINI, 2009). Nessa direção, CoPs têm sido associadas a estratégias de aprendizagem por meio de compartilhamento de conhecimento entre seus membros, inclusive em ambientes de aprendizagem virtuais (COOKCRAIG; SABAH, 2009; NEUFELD; FANG; WAN, 2013).

A partir de 2000, CoPs começaram a ser pesquisadas como instrumentos de gestão organizacional para alcance de efetividade de trabalhos em grupo (BETTIOL; SEDITA, 2011; BRAITHWAITE et al., 2009; CHU; KHOSLA, 2009) ou como estratégia de comunicação organizacional (BELL; LAl; LI, 2012). Autores como Chiu, Hsu e Wang (2006) identificaram que a adoção de CoPs gerou à empresa por eles investigada cerca de $200 \%$ de retorno sobre o investimento (ROI), em função da realização de projetos conjuntos de desenvolvimento de softwares com colegas de várias partes do mundo

As comunidades de prática também têm sido investigadas como instrumento de gestão do conhecimento, como mecanismos que permitem a geração e disseminação do conhecimento tácito (ARDICHVILI, et al., 2006), como estratégia para compartilhamento de conhecimento (JEON; KIM; KOH, 2011; KIM, HONG; SUH, 2012), ou para transformação de conhecimento novo em conhecimento utilizável (CADIZ; SAWYER; GRIFFITH, 2009). Esses estudos mostram que as CoPs são pesquisadas para além do contexto de aprendizagem, e destacam a sua relevância no campo da gestão organizacional e em áreas correlatas. Em práticas corporativas, comunidades de prática nem sempre são denominadas como tal. Elas são chamadas, por exemplo, de comunidades de aprendizagem" (Hewlett-Packard Company), "grupos familiares" (Xerox Corporation), "grupos temáticos" (Banco Mundial), "grupos de pares" (British Petroleum), "redes de conhecimento" na IBM Global Services (GONGLA; RIZZUTO, 2001), dentre outras denominações encontradas.

A literatura apresenta igualmente o surgimento de comunidades de prática virtuais com objetivos específicos. Um exemplo são as ENoPs (Electronic Networks of Practice), comunidades online que se formam em torno de um tema com objetivo de ajuda mútua (TEIGLAND; WASKO, 2004). Mais recentemente, mencionam-se as MCoPs (Mobile Communities of Practice) (KIETZMANN et al., 2013), cuja conectividade móvel pode promover o compartilhamento de conhecimento e trabalhos colaborativos por meio de iPad ou Android, com uso igualmente de realidade imersiva (MAJEWSKI; USORO; KHAN, 2011).

Face à associação existente entre o compartilhamento de conhecimento e a criação de conhecimento novo (DU PLESSIS, 2007; SAENZ; ARAMBURU; BLANCO, 2012), e deste com a inovação (HANA, 2013; QUINTANE et al., 2011), as CoPs também têm sido consideradas facilitadoras de processos de inovação. Por exemplo, Mork, Hoholm e Aanestad (2006) relatam como a adoção de novos procedimentos cirúrgicos foi possibilitada com o auxílio de VCoPs.

A essência comum que acompanha o conceito de CoPs nas suas transformações pode ser descrita por características cunhadas pelos autores seminais: CoPs constituem-se de pessoas, reunidas em torno de um domínio de interesse e que discutem práticas nesse domínio (WENGER; MCDERMOTT; SNYDER, 2002), com engajamento mútuo, empreendimento conjunto e práticas compartilhadas (WENGER, 1998).

\subsection{Definições para "comunidade de prática" - compreensões até 2014}

Embora o termo "comunidade de prática" tenha sido cunhado por Lave e Wenger na década de 90, a ideia em si não é nova: "desde os tempos das cavernas, os homens se reuniam

Perspectivas em Gestão \& Conhecimento, João Pessoa, v. 8, número especial, p. 102-117, out. 2018. 
em volta das fogueiras para discutirem estratégias de caça, ou para compartilharem conhecimentos adquiridos sobre novas frutas comestíveis" (WENGER; MCDERMOTT; SNYDER 2002 , p. 5). Em outras épocas da história, alfaiates repassavam seus conhecimentos às novas gerações ao se reunirem com grupos de aprendizes, que a partir da experiência dos veteranos e da observação das suas práticas, absorviam as técnicas de execução de determinada atividade, bem como aspectos da cultura compartilhada no grupo (LAVE; WENGER, 1991), ou seja, de aprendizagem situada, contextualizada em situação prática. Nessa perspectiva, CoP pode ser compreendida como "um conjunto de relações entre pessoas, atividades e mundo, [...] tangenciado por outras comunidades de prática" (MENDES; URBINA, 2015, p. 308).

A definição de Lave e Wenger (1991) tem sido empregada predominantemente por autores da área da educação, como exemplo Ardichvili (2008) e Nistor, Schworm e Werner (2012). Em 1998, Wenger define CoP como um grupo que pode ser constituído por profissionais unidos informalmente por um propósito comum. $O$ autor passa a dar atenção para o contexto de organizações, no qual o indivíduo visa à aprendizagem para fins profissionais. O autor explicita que uma CoP é uma entidade de pessoas unidas por três dimensões: engajamento mútuo, empreendimento conjunto e repertório compartilhado. $O$ engajamento mútuo representa a interação entre os membros que leva à criação de um sentido ou de significado compartilhado do grupo. O empreendimento conjunto é o trabalho que visa a um objetivo comum. O repertório compartilhado são os recursos, os jargões e os termos comuns que servem de meios de negociação entre os membros (Ll et al., 2009).

Já em 2000, Wenger e Snyder definem CoP como "um grupo de pessoas informalmente ligadas por expertises compartilhadas e paixão por um empreendimento conjunto" (p.139). Empresas como a IBM alicerçaram suas comunidades de prática com base nessa definição (GONGLA; RIZZUTO, 2001).

Em 2002, Wenger, McDermott e Snyder definem CoP como "um grupo de pessoas que compartilham um interesse, um rol de problemas ou uma paixão em um tema, e que aprofundam o conhecimento e expertise sobre ele a partir de interações regulares" (p.4). Na obra desses autores, intitulada "Cultivating Communities of Practice - a Guide to Managing Knowledge", é expressa a aplicação da aprendizagem social como diferencial competitivo para organizações, na qual se aborda o emprego de estruturas sociais (comunidades) como instrumentos de gestão. Essa definição é citada com frequência em pesquisas na área de saúde (BRAITHWAITE et al., 2009; SORENSEN, DUBOIS; PATON, 2012; MENDIZABAL, SOLINÍS; GONZÁLEZ, 2013) com foco em CoPs como instrumentos de melhoria de práticas existentes e de inovações na medicina. Outro campo do conhecimento que emprega essa definição é a área de gestão de negócios. Exemplos incluem trabalhos de Chu, Khosla e Nishida (2012); Lin e Lee (2012); Probst e Borzillo (2008) e Theodorakopoulos, Preciado e Bennett (2012). Esses autores apresentam estudos empíricos em organizações com foco predominante na melhoria de desempenho e na competitividade.

Desde 2002, diferentes autores propuseram novos elementos à definição de CoP. Se no contexto inicial de ensino-aprendizagem situada as comunidades de práticas eram compreendidas como grupos de surgimento espontâneo (WENGER, 1998), trabalhos recentes sugerem a criação e o cultivo de CoPs com objetivos claros e direcionados por organizações, como um instrumento de gestão do conhecimento, e esse entendimento é o que mais atende às necessidades do contexto empresarial de negócios (WEISSENBERG; EBERT, 2011; JEON; KIM; KOH., 2011). Uma das razões do interesse de corporações pelas comunidades de prática é sugerida por Hildreth e Kimble (2004): no cenário atual de mudanças constantes e rápidas, as organizações buscam estruturas mais fluidas e flexíveis que as tradicionais formas baseadas em hierarquias funcionais, que Ihes permitam improvisar soluções rápidas. A utilização da definição de CoP no campo empresarial, ocorreu, segundo Van Baalen, Boemenhof-Ruwaard e

Perspectivas em Gestão \& Conhecimento, João Pessoa, v. 8, número especial, p. 102-117, out. 2018. 
Van Heck (2005), por iniciativa de Brown e Duguid (2000), que se tornaram referências na área de gestão de negócios (JEON; KIM; KOH., 2011).

Bertone et al. (2013) mencionam, a partir de uma análise de publicações sobre CoPs, a existência de duas grandes vertentes: as CoPs "de facto" e as CoPs instrumentais. Nas primeiras enfatiza-se a existência de um domínio e de práticas compartilhados, de um senso de comunidade e de significados e propósitos conjuntos (WENGER; MCDERMOTT; SNYDER 2002). Nas segundas, percebem-se menos vínculos com os aspectos teóricos estudados na academia (BERTONE et al., 2013). Na vertente de CoPs instrumentais, configurações organizacionais que se autodenominam CoPs são estudadas quanto à sua efetividade na gestão organizacional, em visão utilitária da gestão do conhecimento. Dentre exemplos de CoPs instrumentais incluem-se grupos denominados de "comunidades de prática" em corporações, a exemplo da Xerox (Allee, 2000), IBM (GONGLA; RIZZUTO, 2001), Shell (MCDERMOTT, 2000) e Caterpillar (ARDICHVILI et al. (2006), com configurações e propósitos diferenciados.

Penfold (2010) esclarece que no contexto educacional, as CoPs enfatizam o papel da aprendizagem mútua por meio das interações entre pessoas, ao passo que no contexto empresarial, a ênfase é na resolução de problemas, na realização de tarefas e na congregação de pessoas em torno de um objetivo da organização.

A análise das definições e dos contextos de utilização das CoPs revela que os seguintes elementos são comuns na caracterização de CoPs em estudo identificados, na literatura selecionada, até 2014: informalidade nas relações, voluntariedade na adesão, regularidade de interações, e intencionalidade de aprendizagem pessoal ou de resolução de problema na organização. Nesse contexto, com base na revisão sistemática da literatura multidisciplinar realizada até aquele ano, Wilbert (2015) propôs a definição:

\begin{abstract}
Comunidade de prática é um grupo formado por pessoas que informal e voluntariamente se unem para compartilharem conhecimentos sobre um domínio e discutem problemas, paixões e interesse sobre ele em interações regulares visando ao aumento de sua própria competência por meio da aprendizagem situada ou para resolver problemas vinculados ao domínio, para atingir objetivos da organização (WILBERT, 2015, p.54).
\end{abstract}

Passados alguns anos, o presente artigo adota essa definição como ponto de referência para investigar a existência de novas alterações de compreensão do conceito de CoP a partir de então, conforme explanado na próxima seção.

\title{
3 PROCEDIMENTOS METODOLÓGICOS
}

Para o presente artigo, em continuidade à pesquisa realizada até 2014 por Wilbert (2015), realizou-se uma atualização da revisão da literatura a partir desse tempo. Dessa forma, revisitou-se a literatura em setembro de 2017 a partir de revisão sistematizada na base Scopus, com a expressão "communit* of practice" em título de artigos e periódicos, para o período 2014-2017. Esse critério foi empregado com base no entendimento que, artigos que explicitam "comunidade de prática" no título, apresentam maiores probabilidades de trazerem a definição do construto no corpo da publicação. A partir do retorno de 401 documentos na base Scopus, foram destacados os 50 artigos mais relevantes, os 50 mais atuais e os 50 mais citados no período considerado. Uma busca adicional foi realizada na base Scielo, com vistas a integrar pesquisas nacionais. Utilizou-se a query "comunidade de prática" OR "comunidades de prática" no período de 2014 a 2017, com obtenção de retorno de 21 publicações. Após eliminadas as duplicidades e selecionados os artigos com acesso na íntegra, o conjunto de trabalhos considerados compôs-se de 52 artigos que trazem em seu corpo uma definição de

Perspectivas em Gestão \& Conhecimento, João Pessoa, v. 8, número especial, p. 102-117, out. 2018. 
comunidade de prática. Dois outros artigos foram acrescentados (OMIDVAR; KISLOV, 2014; FARNSWORTH, KELANTOHU; WENGER-TRAYNER, 2016) por explicitarem diretamente a transformação conceitual de comunidade de prática, o que contribui de forma expressiva ao presente trabalho. Assim, o portfolio final compôs-se de 54 artigos, dos quais 48 empíricos. 0 tratamento dos dados foi realizado de forma manual, com o emprego de planilha Excel. A categorização dos dados baseou-se em aspectos que caracterizam CoPs (LAVE; WENGER, 1991; WENGER, MCDERMOTT; SNYDER, 2000; WILBERT, 2015). Dessa forma, os dados dos estudos empíricos selecionados foram analisados quanto a: a) expressão empregada pelos autores para definirem CoP (grupo de pessoas, processo, espaço, entidade, rede); b) área do conhecimento ; c) gênese da CoP (espontânea ou iniciada pela organização); d) adesão de membros (voluntária ou mandatória); e) objetivo da comunidade; f) regularidade nas interações de membros; g) percepção de paixão pelo domínio por parte dos membros; $h$ ) existência de engajamento mútuo, empreendimento conjunto e repertório compartilhado entre os membros; i) forma de interação (presencial ou virtual), j) percepção de senso de pertencimento; k) percepção de senso de colaboração.

Na seção a seguir apresentam-se os resultados e as análises dos dados.

\section{RESULTADOS E DISCUSSÕES}

\subsection{Artigos sobre CoP a partir de 2014}

Relembra-se que a transformação do conceito de CoP até 2014 encontra-se explicitada na seção 2.1, e que por esse motivo, neste artigo apresentam-se os resultados a partir daquele ano.

No período 2014-2017, nos 48 estudos empíricos, constatou-se que os objetivos para a criação e manutenção de CoPs estão relacionados à aprendizagem individual (39\%), ao compartilhamento do conhecimento (31\%) e ao atendimento explícito de objetivos organizacionais (30\%) (Figura 3).

Figura 3 - Objetivos de CoPs em organizações (2014 - 2017)

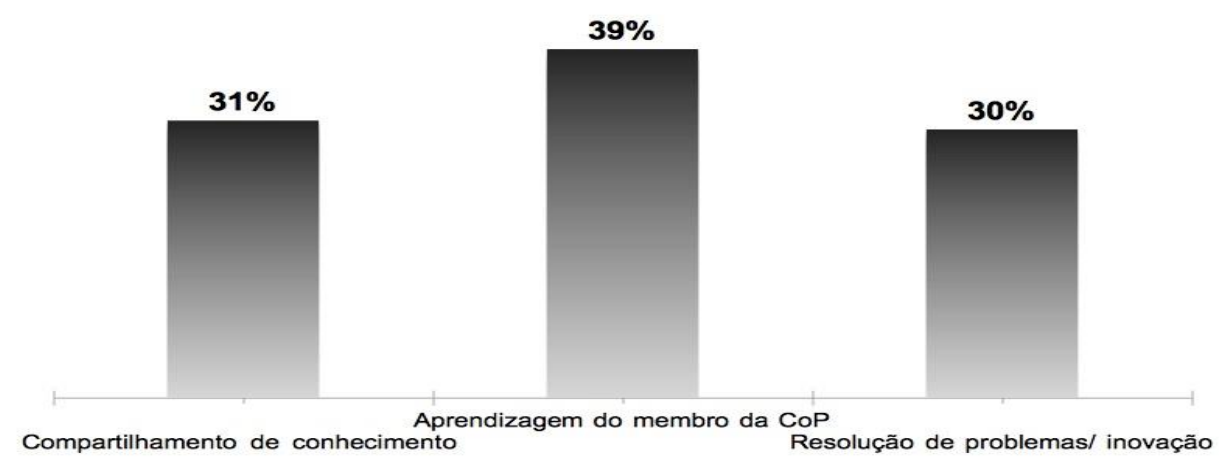

Fonte: Autoras, 2017

Com relação ao contexto em que se inserem as publicações, dos 48 artigos, $67 \%$ mencionam a aprendizagem e $31 \%$ tratam de gestão, o que sugere que o pensamento original de aprendizagem situada de Lave e Wenger (1991) continua a representar, após 25 anos, a principal razão da existência de CoPs em organizações nos estudos analisados. A perspectiva do emprego de CoPs como forma de gestão com vistas à melhoria de desempenho, resolução de problemas e de busca pela inovação, sinaliza que a visão instrumental de CoPs continua a

Perspectivas em Gestão \& Conhecimento, João Pessoa, v. 8, número especial, p. 102-117, out. 2018. 
ocupar um espaço significativo no universo das pesquisas sobre comunidades de prática. Todavia, a visão instrumental de gestão organizacional é menos evidente em pesquisas nacionais, que privilegiam a questão da aprendizagem situada (MENDES; URBINA, 2015).

Nos artigos selecionados, uma associação entre a forma como uma CoP surge (espontânea ou direcionada por uma organização), e a forma de adesão de membros (voluntária ou obrigatória) nos estudos empíricos, foi identificada nas seguintes combinações:

a) gênese espontânea e adesão voluntária: nesta categoria enquadram-se $26,5 \%$ das CoPs apresentadas nos estudos. Nos trabalhos nacionais escolhidos para este estudo, esse índice é de $40 \%$. Destacam-se as CoPs voltadas para a aprendizagem individual de um novato na área do conhecimento (LANGDON et al., 2014), para o compartilhamento de conhecimento entre atores envolvidos em projetos (FERRAZ; DORNELAS, 2015; BORZILLO, 2017; FOGAÇA; HALU, 2017) e para a melhoria de desempenho de atividades organizacionais, mormente no setor educacional (FERREIRA; DA SILVA, 2014; HOU, 2015; NICHOLAS, 2017).

b) gênese espontânea e adesão necessária: $4 \%$ dos casos relatados tratam de CoPs formadas por pessoas que participam de cursos ou projetos. Por iniciativa de professores, emergem CoPs, com formação de um espaço restrito de interação, de adesão necessária por parte dos alunos. Aquele que não se insere na comunidade acaba por ficar à margem do projeto ou do curso (GONZÁLEZ-HOWARD; MCNEILL, 2016);

c) gênese direcionada e adesão voluntária: essa configuração foi observada em $26,5 \%$ dos estudos selecionados. Tratam-se de organizações que criam CoPs, e disponibilizam infraestrutura (virtual ou não) para compartilhamento de conhecimentos e experiências (SALES; DORNELAS, 2014; HERRERO, 2016; LEWIS, 2017) com a presença de gestão da comunidade pela organização, com vistas a melhorias de desempenho (PATTINSON; PREECE, 2014; LEE et al., 2015; KEITH, SMITH; TAYLOR, 2017).

d) gênese direcionada e adesão necessária: $28,5 \%$ dos estudos tratam de CoPs cuja origem ocorreu por iniciativa da organização (escola, grupo de projeto), com a necessidade de adesão do estudante à CoP para não ser excluído (CYRINO, 2016; MARCOLINO; LOURENÇO; REALI, 2016).

Menciona-se que em $14,5 \%$ dos estudos analisados não foi possível identificar, pelo relato do caso, a configuração com relação a gênese e a adesão de membros da CoP.

Os arranjos anteriores foram identificados em estudos onde o fenômeno pesquisado é apresentado como CoP, e revelam que a ideia original de Lave e Wenger (1991), de surgimento espontâneo e adesão voluntária, sofreram modificações em diferentes contextos. Os artigos analisados sugerem que prevalecem os casos em que a gênese é promovida pela organização com a necessária adesão dos membros. Essa condição ocorre sobretudo no contexto de educação, onde o aluno necessita participar de uma determinada CoP para poder participar do curso. Contudo, mesmo nos casos em que a obrigatoriedade se dá com objetivo de uma aprendizagem mais efetiva (NISTOR et al., 2014), os relatos sugerem que a informalidade no funcionamento das CoPs é uma característica que se apresenta na quase totalidade dos estudos analisados. Relembra-se Wenger (1998): a fluidez face a horizontalidade nas relações e a presença de hierarquia no nível mínimo de gestão da comunidade como atributo intrínseco a CoPs ratificam-nas como alternativa para se transcender amarras hierárquicas. Mendes e Urbina (2015) advogam que "os aspectos informais dos relacionamentos para produção de conhecimentos não-canônicos" (p. 323) são características que distinguem CoPs de grupos ou de equipes de trabalho.

Alguns pesquisadores (MARCOLINO; LOURENÇO, 2016) mencionam o fator "regularidade" nas interações como característica de CoPs. Dos estudos selecionados para esta pesquisa, 75,5\% explicitam a existência de interações regulares entre os membros das CoPs como forma de promover a proximidade, a informalidade e a horizontalidade nas relações, mesmo em VCoPs (TSENG ; KUO, 2014).

Perspectivas em Gestão \& Conhecimento, João Pessoa, v. 8, número especial, p. 102-117, out. 2018. 
Os dados revelam ainda que no período de 2014-2017, 53\% das CoPs tratam-se de comunidades presenciais, e sinaliza que o conceito de CoP não está necessariamente vinculado a tecnologia. Um outro aspecto mencionado em CoPs por Wenger (2002) é a existência de paixão ou interesse por um domínio. Os estudos de 2014 a 2017 evocam "interesse" no domínio por parte de membros, e o atributo "paixão" menos perceptível em $64 \%$ dos estudos selecionados. Contudo, registre-se que mesmo nos casos em que a adesão ocorre por "necessidade" (por exemplo, participar de curso ou de projeto), tal fato não exclui "paixão" (CHO, 2016) ou grande interesse (CYRINO, 2016; MARCOLINO; LOURENÇO, 2016).

Ainda, os resultados indicam que o mosaico de variações em torno do conceito de CoP nos estudos selecionados distancia-se de uma convergência: $6 \%$ não explicitam definição de CoP, ainda que o construto seja parte dos estudos (LEWIS, 2017; OMIDVAR; KISLOV, 2014).

Quando explicitada, a definição de CoP é apresentada como:

a) um grupo de pessoas - dentre os artigos pesquisados, $68 \%$ apresentam uma visão antropocêntrica, com ênfase em seus estudos no aspecto "pessoas" como centro das atenções de suas pesquisas. Para os que compartilham dessa visão, CoPs são pessoas reunidas em torno de um propósito, individual ou coletivo.

b) uma rede, um processo ou um sistema de relações - para $20 \%$ dos artigos pesquisados, CoPs são as relações que emergem das interações de pessoas, para fins de aprendizagem situada ou para alcance de fins organizacionais.

c) um espaço, um contexto ou uma estrutura (presencial ou virtual) que permite interações horizontais - $12 \%$ das pesquisas acessadas enfatizam o aspecto do lócus de compartilhamento de conhecimento que a CoP propicia.

Diante de diversas interpretações sobre o conceito de CoP, uma pergunta adicional emergiu durante a análise dos trabalhos: que elementos embasaram os autores, tanto de estudos teóricos, como de pesquisas empíricas, para caracterizarem um grupo como CoP? Foram identificados os seguintes aspectos:

a) em $69 \%$ dos estudos são perceptíveis "senso de pertencimento" e de "identidade" por parte dos membros como característica que identifica que o grupo é uma CoP; alguns estudos, como Lewis (2017) e Nistor et al. (2014) sugerem a existência de engajamento ou senso de identidade, inclusive membros de CoPs mediadas por tecnologia.

b) $85 \%$ dos estudos sugerem a existência ou indícios de colaboração entre os membros das CoPs estudadas empiricamente, ou mencionam colaboração como atributo de CoPs.

c) três dimensões de CoP descritas por Wenger (1998): o engajamento mútuo, o empreendimento conjunto e o repertório compartilhado, são explicitados como elementos centrais do conceito em $55 \%$ dos estudos.

Lave e Wenger (1991), Wenger (1998) e Wenger, Snyder e McDermott (2002) permanecem na atualidade como os autores basilares em estudos sobre CoPs, mesmo com as adaptações contextuais que o construto sofreu ao longo do tempo. Mendes e Urbina (2015) constataram em sua pesquisa, que Wenger et al. (2002), Lave e Wenger (1991) e Wenger (1998) estão entre os autores mais empregados por estudiosos brasileiros que investigam CoPs, de diferentes áreas do conhecimento.

Etienne Wenger, atualmente Etienne Wenger-Trayner, tem acompanhado o construto e sua transformações no tempo, e ratifica que CoP é um conceito altamente dependente de contexto, e portanto, passível de alterações. Os principais pontos colocados por WengerTrayner em 2014 (OMIDVAR; KISLOV) e em 2016 (FARNSWORTH; KLEANTHOUS; WENGERTRAYNER) são trazidos no próximo tópico. Conforme mencionado em procedimentos metodológicos, por ocasião da atualização da revisão da literatura, identificaram-se dois artigos com relatos da transformação da visão de Etienne Wenger sobre a definição de CoP por ele cunhada na década de 90 . Pela relevância ao propósito do presente trabalho, esses artigos são mencionados na sequência.

Perspectivas em Gestão \& Conhecimento, João Pessoa, v. 8, número especial, p. 102-117, out. 2018. 


\subsection{Transformação Conceitual de CoP segundo Wenger-Trayner}

Omidvar e Kislov (2014) afirmam que nos últimos vinte anos "a teoria de comunidades de prática evoluiu e expandiu em numerosas direções, e reflete a flexibilidade de interpretações na abordagem e sua popularidade através das disciplinas e setores [de atuação]" (p. 267, tradução nossa). No artigo The Evolution of the Communities of Practice Approach: Toward Knowledgeability in a Landscape of Practice-An Interview with Etienne Wenger-Trayne, Omidvar e Kislov (2014) relatam a entrevista com Etienne Wenger-Trayner, que destacou os seguintes aspectos:

a) a relevância de CoPs está na abordagem de que a aprendizagem é um fenômeno que decorre da interação social, e a estrutura de CoP é a estrutura social mais simples que oferece condições de interações de aprendizagem entre o indivíduo e a estrutura social. Nessa linha, "se pessoas aprendem juntas, o resultado é uma comunidade de prática" (WENGERTRAYNER entrevistado por Omidvar e Kislov, 2014, p. 269).

b) o grande ponto de inflexão conceitual de CoPs foi a transformação de um conceito analítico para um conceito instrumental, onde se descobriu a utilidade do conceito para aplicação na prática.

c) há críticas da academia com relação à instabilidade e plasticidade do conceito de CoP; Wenger-Trayner vislumbra que está a emergir uma terceira fase da assim chamada "teoria" de CoP.

d) o conceito de CoP é passível de ser adotado em contextos variados, porém, a essência permanece no fato de que se trata da capacidade social de aprendizagem dos indivíduos.

No artigo de Farnsworth, Kleanthous e Wenger-Trayner (2016), intitulado Communities of practice as a social theory of learning: A conversation with Etienne Wenger, este último enfatiza que CoP "não é um grupo de pessoa per se" (p. 144), mas sim "um processo social de negociação de competências em um domínio ao longo do tempo (idem). Tal processo estrutura relações em que emergem as características constituintes de comunidade de prática, cujo cerne é o domínio. Wenger-Trayner (2016) argumenta que empreendimento conjunto também pode ocorrer em equipes, que não são necessariamente CoPs. É em torno de um domínio que ocorrem as interações, o engajamento mútuo e a criação de repertórios compartilhados.

As colocações de Wenger-Trayner sugerem que o conceito de CoP apresenta tal plasticidade por apresentar elementos inerentes às interações humanas e a sua capacidade de trabalhar o conhecimento. A atividade de criar e transformar conhecimentos ocorre em todos os contextos onde pessoas se reúnem para trocar ideias, transmiti-las ou buscar por algo diverso do existente.

\subsection{Comunidade de Prática: o domínio como cerne na geração de repertório compartilhado, engajamento mútuo e empreendimento conjunto}

O presente estudo observa que, à medida em que comunidades de prática encontram uso em outros contextos, seu conceito se lhes adapta. Porém, faz-se uma ressalva no sentido de alertar para que tais adequações não descaracterizem as CoPs enquanto elas mesmas. Nesse sentido, os trabalhos iniciais de Wenger (1998), com a explicitação das características de uma CoP apresentados na revisão da literatura (empreendimento conjunto, repertório compartilhado e engajamento mútuo), são clássicos norteadores que preservam a identidade de CoPs. Wenger-Trayner (2016) destaca o domínio da comunidade de prática como o atrator para que um grupo de pessoas se relacionem de forma cooperativa, o que reforça a definição

Perspectivas em Gestão \& Conhecimento, João Pessoa, v. 8, número especial, p. 102-117, out. 2018. 
apresentada por Wenger, McDermott e Snyder (2002), explicitada na revisão de literatura deste artigo.

A partir dos trabalhos elencados para esta pesquisa, o conceito proposto por Wilbert (2015) sugere continuar, para o momento, pertinente para CoPs em contexto de organizações. Os artigos pesquisados nos anos posteriores sugerem que a visão instrumental da CoP tem proliferado, como meio de otimizar processos de aprendizagens de alunos em cursos, de melhorar desempenho de projetos ou de resolver problemas organizacionais. Ainda assim, a adesão voluntária pelos indivíduos predominam nos estudos selecionados, e a informalidade na dinâmica de funcionamento faz-se perceptível na maioria das descrições de CoP analisadas. Tal conclusão é igualmente corroborada por Mendes e Urbina (2015), que entendem que os aspectos informais caracterizam CoPs, ainda que atreladas a interesses organizacionais. A paixão pelo domínio não parece ser determinante; porém, um interesse motivado pela vontade de atingir objetivos de aprendizagem individual ou organizacionais mostra-se necessário para que membros atuem nas comunidades. Nos estudos selecionados, ainda que tenham sido identificados diversos entendimentos sobre CoPs, três elementos sugerem ser relevantes para o conceito de CoP: repertório compartilhado, engajamento mútuo e empreendimento conjunto (WENGER, 1998), e o domínio de CoPs é o ponto de convergência de pessoas e processos de interação entre elas (WENGER-TRAYNER, 2016).

\section{CONSIDERAÇÕES FINAIS}

Diante da diversidade de conceitos para a expressão "comunidade de prática" (CoP) e "comunidade de prática virtual" (VCoP), as autoras propuseram neste estudo apresentar os múltiplos entendimentos.

Com origem em um conceito analítico da área da educação, com Lave e Wenger no início da década de 90, CoPs ganham espaço nas organizações como ferramenta ou estratégia de gestão do conhecimento e de gestão organizacional. Assim, aspectos antes não considerados, como a criação de CoPs pela organização ou a sua gestão baseada em objetivos a serem alcançados, passam a ser aceitos dentro desta abordagem, ainda que não isenta de polêmicas. As autoras deste artigo entendem que há elementos centrais na sua concepção: a existência de um domínio como núcleo de atração de pessoas, voluntariedade na adesão, informalidade no funcionamento, existência de empreendimento conjunto, engajamento mútuo e repertório compartilhado. A pesquisa sinalizou igualmente uma adoção gradual da mediação tecnológica, de forma que cada vez mais as CoPs serão sinônimos de VCoPs, e o adjetivo "virtual" passa a não acompanhar o termo CoP como um diferencial. Entretanto, a partir de 2014, as pesquisas indicam que o interesse por comunidade presenciais continua significativo. Isso pode significar que CoPs ganham maior relevância na sua dimensão antropocêntrica do que na tecnológica.

O estudo contribui para a compreensão de que o conceito de CoP surgiu em um contexto de aprendizagem situada, e, decorrente de seu significado mais intrínseco estar relacionado com interações de pessoas, tornou-se apropriado para ser utilizado em outros contextos. Dessa forma, neste estudo foi mostrado que definições podem se transformar, e nem por isso o conceito original perde sua validade para determinados contextos.

Sugere-se estudos mais aprofundados sobre a temática, à luz de uma análise semântica dos conceitos emergentes de CoPs/VCoPs associado a padrões, caso existam, a disciplinas da ciência, de certa forma já sugerido por Mendes e Urbina (2015). Adicionalmente, é oportuna investigar a existência de escolas e de tendências de evoluções do conceito ora em discussão, à medida em que a sociedade se modifica. E periodicamente atualizar esta pesquisa: afinal, conceitos antropocêntricos são passíveis de adaptações contextuais e temporais, e certamente CoP é um deles.

Perspectivas em Gestão \& Conhecimento, João Pessoa, v. 8, número especial, p. 102-117, out. 2018. 


\section{REFERÊNCIAS}

ARDICHVILI Alexandre; MAURER, Martin, LI Wei; WENTLING, Tim.; STUEDEMANN, Reed. Cultural influences on knowledge sharing through online communities of practice. Journal of Knowledge Management, v. 10, n. 1, p. 94-107, 2006.

ARDICHVILI, Alexandre. Learning and Knowledge Sharing in Virtual Communities of Practice: Motivators, Barriers, and Enablers. Advances in Developing Human Resources, v. 10, n.4, p. 541-554, 2008.

BELL, Geoffrey G.; LAI, Fujun; LI, Dahui. Firm orientation, community of practice, and Internetenabled interfirm communication: Evidence from Chinese firms. Journal of Strategic Information Systems, v. 21, p. 201-215, 2012.

BERTONE, Maria P.; MEESSEN, Bruno, CLARYSSE, Guy, HERCOT, David, KELLEY, Allison, KAFANDO, Yamba, LANGE, Isabelle, PFAFFMANN, Jerôme, RIDDE, Valéry, SIELEUNOU, Isidore, WITTER, Sophie. Assessing communities of practice in health policy: a conceptual framework as a first step towards empirical research. Health Research Policy and Systems, v.11, n. 13, p.1-13, 2013.

BETTIOL, Marco; SEDITA, Silvia R. The role of community of practice in developing creative industry projects. International Journal of Project Management, v. 29, p. 468-479, 2011.

BORZILLO, Stefano. "Balancing control and autonomy in communities of practice: governance patterns and knowledge in nine multinationals". Journal of Business Strategy, v. 38, n. 3, p. 1020, 2017.

BRAITHWAITE, Jeffrey; WESTBROOK, Johanna I.; RANMUTHUGALA, Geetha; CUNNINGHAM, Frances; PLUMB, Jennifer; WILEY, Janice; BALL, Diane; HUCKSON, Sue; HUGHES, Cliff; JOHNSTON, Brian; CALLEN, Joanne; CRESWICK, Nerida; GEORGIOU, Andrew; BETBEDERMATIBET, LUC; DEBONO, Deborah. The development, design, testing, refinement, simulation and application of an evaluation framework for communities of practice and socialprofessional networks. BMC Health Services Research, v.9, p. 162-170, 2009.

BROWN, John S.; DUGUID, Paul. Knowledge and Organization: A Social-Practice Perspective. Organization Science, v. 12, n. 2, p.198-213, 2001.

CADIZ, David.; SAWYER John E.; GRIFFITH, Terri L. Developing and Validating Field Measurement Scales for Absorptive Capacity and Experienced Community of Practice. Educational and Psychological Measurement, v. 60, n. 6, p. 1035-1058, 2009.

CHIU, Chao-Min; HSU, Meng-Hsiang; WANG, Eric T.G. Understanding knowledge sharing in virtual communities: an integration of social capital and social cognitive theories. Decision Support Systems, v. 42, p. 1872-1888, 2006.

$\mathrm{CHO}$, Hyesun. Under co-construction: An online community of practice for bilingual pre-service teachers. Computers \& Education, v. 92-93, p. 76-89, 2016.

$\mathrm{CHU}$, Mei-Tai; KHOSLA, Rajiv. Index evaluation and business strategies on communities of practice. Expert Systems with Applications, v. 36, p. 1549-1558, 2009.

CHU, Mei-Tai.; KHOSLA; Rajiv.; NISHIDA, Toyoaki. Communities of practice model driven knowledge management in multinational knowledge based enterprises. Journal of Intelligent Manufacturing, v. 23, n. 5, p. 1707-1720, 2012.

Perspectivas em Gestão \& Conhecimento, João Pessoa, v. 8, número especial, p. 102-117, out. 2018. 
COOK-CRAIG, Patricia G.; SABAH, Yekoutiel. The role of virtual communities of practice in supporting collaborative learning among social workers. British Journal of Social Work, v. 39, p. 725- 739, 2009.

CORSO, Mariano; GIACOBBE, Andreas; MARTINI, Antonella. Designing and managing business communities of practice. Journal of Knowledge Management, v. 13, n. 3, p. 73 - 89, 2009.

CYRINO, Márcia C. C. T. Desenvolvimento da Identidade Profissional de Professores de Matemática em Comunidades de Prática: Reificações do Ensino do Raciocínio Proporcional. Bolema, v. 30, n. 54, p.165-187, 2016.

DU PLESSIS, Marina. The role of knowledge management in innovation. Journal of Knowledge Management, v. 11, n.4, p. 20-29, 2007.

FARNSWORTH, Valeria; KLEANTHOUS, Irene; WENGER-TRAYNER, Etienne. Communities of practice as asocial theory of learning: A conversation with Etienne Wenger. British Journal of Educational Studies, v. 64, n. 2 , p. 139-160, 2016.

FERRAZ, Isabela N.; DORNELA, Jairo S. Repertório Compartilhado de Recursos em Comunidades Virtuais de Prática: um estudo dos mecanismos de interação, organização e controle em grupos de pesquisa científica. 0 \& S, v. 22, n. 72, p.100-122, 2015.

FERREIRA, Andréia A.; DA SILVA, Bento D. Comunidade de prática on-line: Uma estratégia para o desenvolvimento profissional dos professores de história. Educação em Revista, v. 30, n. 1, p. 37-64, 2014.

FOGAÇA, Francisco C.; HALU, Regina C. Comunidades de prática e construção identitária de formadores de professores em um programa de formação continuada. RBLA, v. 17, n. 3, p. 427-454, 2017.

GONGLA, Patricia; RIZZUTO, Christina R. Evolving communities of practice: IBM Global Services. IBM Systems Journal, v. 40, n. 4, p.842-862, 2001.

GONZÁLEZ-HOWARD; Maria; MCNEILL, Katherine L. Learning in a Community of Practice: Factors Impacting English-Learning Students' Engagement in Scientific Argumentation. Journal of Research in Science Teaching, v. 53, n. 4, p. 527-553, 2016.

HANA, Urbancová. Competitive Advantage Achievement through Innovation and Knowledge. Journal of Competitiveness, v. 5, n. 1, p. 82-96, 2013.

HERRERO, Carmen. The Film in Language Teaching Association (FILTA): a multilingual community of practice. ELT Journal, v. 70, n. 2, p. 190-200, 2016.

HILDRETH, Paul; KIMBLE, Chris. Knowledge Networks: innovation through communities of practice. Idea Group Publishing: Hershey, London, Melbourne, Singapore, 2004, 357p.

HOU, Hong, What makes an online community of practice work? A situated study of Chinese student teachers' perceptions of online professional learning. Teaching and Teacher Education v. 46, p. $6-16,2015$.

JEON, Su-Hwan; KIM Young-Gul; KOH Joon. Individual, social, and organizational contexts for active knowledge sharing in communities of practice. Expert Systems with Applications, v. 38, p. 12423-12431, 2011.

KEITH, Brian W.; SMITH, Bonnie. J.; TAYLOR, Laurie. N. Building a Collaborative Position Description Archive as a Community of Practice. Building a Collaborative Position Description Archive as a Community of Practice. Libraries and Academy, v. 17, n. 2, p. 419-434, 2017.

Perspectivas em Gestão \& Conhecimento, João Pessoa, v. 8, número especial, p. 102-117, out. 2018. 
KIETZMANN, Jan; PLANGGER, Kirk; EATON, Ben; HEILGENBERG, Kerstin; PITT, Leyland; BERTHON, Pierre. Mobility at work: a typology of mobile communities of practice and contextual ambidexterity. Journal of Strategic Information Systems. v.22, p. 282-297, 2013.

KIM, Sung-jin.; HONG, Jong-yi.; SUH, Eui-ho. A diagnosis framework for identifying the current knowledge sharing activity status in a community of practice. Expert Systems with Applications. v. 39, p. 13093-13107, 2012.

LANGDON, Frances J.; ALEXANDER, Patricia A.; RYDE, Alexis; BAGGETTA, Peter. A national survey of induction and mentoring: How it is perceived within communities of practice. Teaching and Teacher Education, v. 44, p. 92-105, 2014.

LAVE, Jane.; WENGER, Etienne. Situated learning: legitimate peripheral participation. Cambridge University Press: New York, 1991. 138p.

LEE, Lorraine; REINICKE, Bryan; SARKAR, Robin; ANDERSON, Rita. Learning Through Interactions: Improving Project Management Through Communities of Practice. Project Management Journal, v. 46, n.1, p. 40-52, 2015.

LEWIS, Steven. Communities of Practice and PISA for Schools: Comparative Learning or a Mode of Educational Governance? Education Policy Analysis Archives, v. 25, n. 92, edição especial, p. 1-25, 2017.

LI, Linda C.; GRIMSHAW, Jeremy M.; NIELSEN, Camilla; JUDD, Maria; COYTE, Peter C.; GRAHAM, Ian D. Use of communities of practice in business and health care sectors: a systematic review. Implementation Science, v. 4, p.11-19, 2009.

LIN, Yu-Cheng; LEE, Hsin-Yu. Developing project communities of practice-based knowledge management system in construction. Automation in Construction, 22, 422-432, 2012.

MAJEWSKI, Grzegorz; USORO Abel, KHAN; Imran. Knowledge sharing in immersive virtual communities of practice". VINE, v. 41, n. 1, p. 41-62, 2011.

MARCOLINO, Taís Q.; LOURENÇO, Gerusa E.; REALI, Aline M. M. R. "This is my take-home for life!": professional practice learning on a Community of Practice. Interface Comunicação Saúde Educação, 2016.

MCDERMOTT, Richard. Community development as a natural step. Knowledge Management Review, v. 3, n. 5, p. 16-19, 2000.

MENDES, Luciano; URBINA, Ligia M. S. Análise sobre a Produção Acadêmica Brasileira em Comunidades de Prática. Revista de Administração Contemporânea, Rio de Janeiro, v. 19, 3a Edição Especial, p. 305-327, 2015.

MENDIZABAL, Galder A.; SOLINÍS, Roberto N.; GONZÁLEZ, Irune Z. HOBE+, a case study: a virtual community of practice to support innovation in primary care in Basque Public Health Service. BMC Family Practice, v.14, n. 168, p. 1-10, 2013.

MORK, Bjorn E.; HOHOLM, Thomas; AANESTAD, Margunn. Constructing, enacting and packaging innovations. European Journal of Innovation Management, v.9, n. 4, p. 444-465, 2006.

NEUFELD, Derrick; FANG, Yulin; WAN, Zeying. Community of Practice Behaviors and Individual Learning Outcomes. Group Decision Negotiation, v.22, p. 617-639, 2013.

NICHOLAS, Celeste R. SciJourn is magic: construction of a science journalism community of practice. Cultural Studies of Science Education, v.12, p. 275-298, 2017.

Perspectivas em Gestão \& Conhecimento, João Pessoa, v. 8, número especial, p. 102-117, out. 2018. 
NISTOR, Nicolae; SCHWORM, Silke; WERNER, Matthias. Online help-seeking in communities of practice: modeling the acceptance of conceptual artifacts. Computers \& Education, v. 59, p. 774-784, 2012.

NISTOR, Nicolae; BALTES, Beate; DASCALU, Mihai; MIHAILA, Dan; SMEATON, George; TRAUSAN-MATU, Stefan. Participation in virtual academic communities of practice under the influence of technology acceptance and community factors. A learning analytics application. Computers in Human Behavior, v. 34, p. 339-344, 2014.

OMIDVAR, Omid; KISLOV, Roman. The Evolution of the Communities of Practice Approach: Toward Knowledgeability in a Landscape of Practice-An Interview with Etienne WengerTrayner. Journal of Management Inquiry, v. 23, n. 3, p. 266-275, 2014.

PENFOLD, Paul. Virtual Communities of Practice: Collaborative Learning and Knowledge Management, Third International Conference on Knowledge Discovery and Data Mining, p. 482-485, 2010.

PROBST, Gilbert; BORZILLO, Stefano. Why communities of practice suceed and why they fail. European Management Journal, v. 26, p. 335-347, 2008.

QUINTANE, Eric; CASSELMAN, R.Mitch; REICHE, Sebastian; NYLUND, Petra A. Innovation as a knowledge-based outcome. Journal of Knowledge Management. v.15, n. 6, p. 928-947, 2011.

SAENZ, Josune; ARAMBURU, Nekane; BLANCO, Carlos E. Knowledge sharing and innovation in Spanish and Colombian high-tech firms. Journal of Knowledge Management, v.16, n. 6, pp. 919-933, 2012.

SALES, Jefferson D. A.; DORNELAS, Jairo S. Ações coletivas e tecnologia da informação: efeitos indutores à configuração dos coletivos inteligentes. Revista de Administração Contemporânea, v.18, n. 4, p. 487-50, 2014.

SORENSEN, Rena; DUBOIS, Roberta; PATON, Patricia L. Innovative Community of practice Development within the Alberta Respiratory Community. Canadian Journal of Respiratory Therapy, v. 48, n. 3, p. 6-11, 2012.

TEIGLAND, Robin; WASKO, Molly M. (2004). Extending richness with reach: participation and knowledge exchange in electronic networks of practice. In: KIMBLE, C.; HILDRETH, P. Knowledge networks: innovation through communities of practice. London: Idea Group, p. 230-242, 2004.

TSENG, Fan-Chuan; KUO, Feng-Yang (2014). A study of social participation and knowledge sharing in the teachers' online professional community of practice. Computers \& Education, v. 72, pp. 37-47, 2014.

THEODORAKOPOULOS, Nicholas; PRECIADO, Deycy J. S.; BENNETT, David. Transferring technology from university to rural industry within a developing economy context: the case for nurturing communities of practice. Technovation, v. 32, p. 550-559, 2012.

VAN BAALEN, Peter; BLOEMHOF-RUWAARD, Jacqueline; VAN HECK, Eric. Knowledge sharing in an emerging network of practice: the role of a knowledge portal. European Management Journal, v. 23, n. 3, p. 300-314, 2005.

WEISSENBERG, M. A.; EBERT, Dominik. Stitching an Organisation's Knowledge Together: Communities of Practice as Facilitator for Innovations Inside an Affiliated Group. In: HOWLETT, R. Innovation through Knowledge Transfer 2010, Springer-Verlag Berlin Heidelberg, p. 245-252, 2011.

Perspectivas em Gestão \& Conhecimento, João Pessoa, v. 8, número especial, p. 102-117, out. 2018. 
WENGER, Etienne C.; SNYDER, William M. Communities of practice: the organizational frontier. Harvard Business Review, January-February, p. 139-145, 2000.

WENGER, Etienne. Communities of Practice: learning, meaning and identity, Cambridge University Press, 1998.

WENGER, Etienne; MCDERMOTT, Richard; SNYDER William M. Cultivating Communities of Practice. A Guide to managing knowledge. Massachussets: Harvard Business Press, 2002.

WILBERT, Julieta K. W. Características de VCoPs que influenciam processos de inovação: estudo de caso em uma empresa publica brasileira. 2015. 213f. Dissertação (Mestrado em Engenharia e Gestão do Conhecimento) - Universidade Federal de Santa Catarina, Florianópolis, 2015.

WHELAN, Eoin. Knowledge Exchange in Electronic Networks of Practice: Toward a Conceptual Framework. International Federation for Information processing (IFIP), 206, The Transfer and Diffusion of Information Technology for Organizational Resilience, eds. B. Donnellan, Larsen T., Levine L., DeGross J. (Boston: Springer), p. 21-31, 2006.

YOUNG, Ronald. (Org.). Knowledge management tools and techniques manual. United Kingdom: APO, 2010.

Artigo recebido em 19/10/2018 e aceito para publicação em 29/10/2018 